\title{
Flying Instability due to Organic Compounds in Hard Disk Drive
}

\author{
Koji Sonoda \\ Advanced Technology Development Department, Storage Products Design and Production Division, \\ Toshiba Corporation, 2-5-1 Kasama, Sakae-ku, Yokohama 247-8585, Japan \\ Correspondence should be addressed to Koji Sonoda, koji4.sonoda@toshiba.co.jp
}

Received 27 July 2012; Accepted 2 December 2012

Academic Editor: Norio Tagawa

Copyright ( 2012 Koji Sonoda. This is an open access article distributed under the Creative Commons Attribution License, which permits unrestricted use, distribution, and reproduction in any medium, provided the original work is properly cited.

The influence of organic compounds (OCs) on the head-disk interface (HDI) was investigated in hard disk drives. The drives were tested at high temperature to investigate the influence of gaseous OC and to confirm if the gaseous OC forms droplets on head or disk. In the experiment, errors occurred by readback signal jump and we observed the droplets on the disk after full stroke seek operation of the drive. Our results indicate that the gaseous OC condensed on the slider and caused flying instability resulting in drive failure due to slider contact with a droplet of liquid OC. Furthermore, this study shows that kinetic viscosity of OC is an important factor to cause drive failure using alkane reagents.

\section{Introduction}

To achieve $1 \mathrm{~Tb} / \mathrm{in}^{2}$ recording density, a head-disk clearance below $2 \mathrm{~nm}$ is required. At this small clearance flying instability, which results in a few nanometers of clearance variation, may cause read/write errors due to the high sensitivity of magnetic recording performance to clearance. In addition to direct contact between head and disk, collision between a lubricant droplet and head may cause vibrations and induce a read/write "signal jump" such as reported by $\mathrm{Li}$ et al. [1]. Fowler and Geiss [2] observed droplets of OC (alkane) on the head that can cause the stiction at the HDI with a visualization setup. With these references, we used model OC instead of using lubricant for accelerated testing to facilitate droplet observation as it is difficult to observe the lubricant droplet on the disk covered with the lubricant film. We found that OC, specifically hydrocarbons, can also make the flying head unstable by contact with a droplet of OC during the write operation, resulting in an unrecoverable read error. In this study, drive level test was carried out to investigate the influence of OC on reliability of head disk interface (HDI).

\section{Experimental Details}

To investigate the influence of OC, we applied $30 \mathrm{mg}$ of hydrocarbon oil to the inside top cover of test drives.
The drives were placed with the top cover down to avoid dropping the hydrocarbon oil to disk. This hydrocarbon oil has a wide distribution of molecular weight from about 100 to 500 grams per mole. For the drive test, several $10000 \mathrm{rpm}$ test drives were built. Tests were conducted at a controlled temperature of $55^{\circ} \mathrm{C}$ to volatilize components of the hydrocarbon oil. The test sequence is shown in Figure 1. First, full stroke seeks between maximum outer radius (OD) and minimum inner radius (ID) without write/read operation (no dynamic flying height control) were conducted for 10 hours. The flying height is about $10 \mathrm{~nm}$ during the full stroke seek. Next, a sequential write operation from OD to ID was conducted. Then, a sequential read operation similar to the sequential write was conducted. The flying height is $3.0 \mathrm{~nm}$ with dynamic flying height control in the sequential write and read operation. If a readback error occurred, Viterbi metric margin (VMM) measurement (read only) near the error position was performed to map the error location. VMM is a function conceived as a means of measuring signal quality during Viterbi decoding. The margin means the difference between the actually received data path (continuous 0/1 data) and ideal path. VMM correlates with sector error rate (SER). When the VMM is larger, SER is worse. The details of VMM measurement are shown in [3]. 


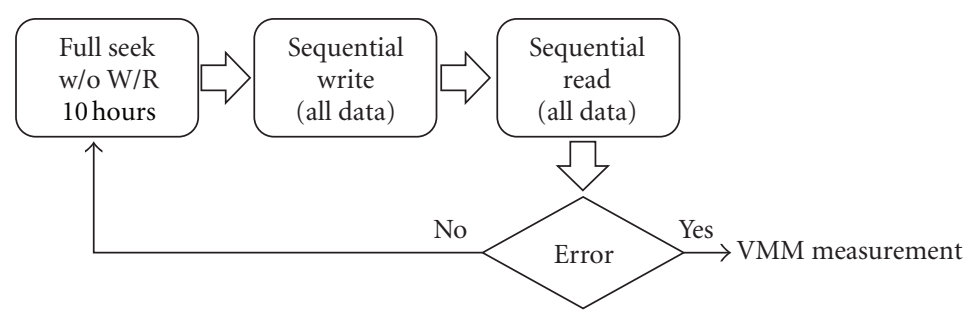

Max seek velocity during full seek operation

$\sim 2.7 \mathrm{~m} / \mathrm{s}$ at middle radius

FIGURE 1: Sequence of drive test accelerated by OC. The fly condition is passive (no dynamic flying height control) during the full stroke seek operation without write/read operation.

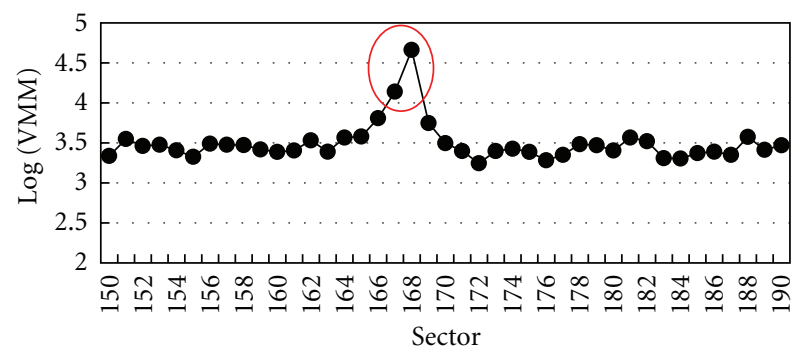

FIGURE 2: VMM changes near the error position.

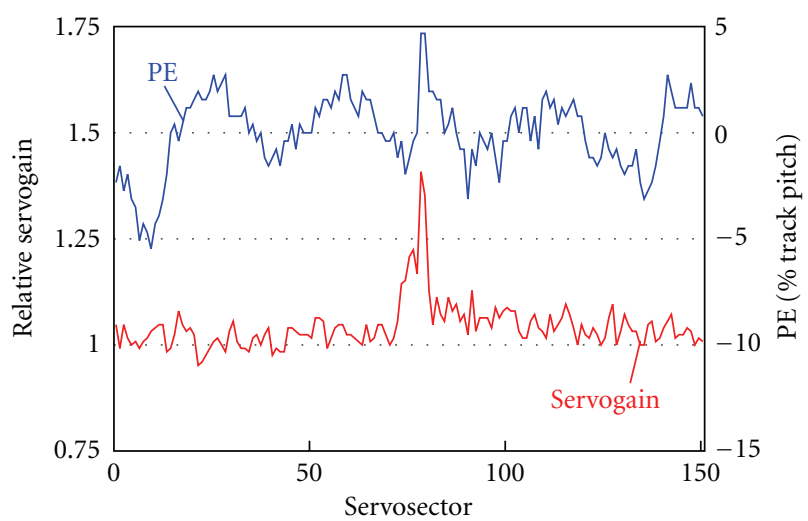

FIGURE 3: Relative servogain and position error (PE) changes when head may contact droplets.

We also tested drives where $100 \mu \mathrm{L}$ of alkane (paraffin or saturated hydrocarbon) solutions were injected into the inside of the drive enclosure on the base casting near the motor to understand what component of the OC causes the error. The test sequence is the same as the above test shown in Figure 1. Table 1 shows the alkanes we used for test and amount of volume/weight loaded into the drives. The weight of icosane, pentacosane, and triacontane excludes hexane's weight. In addition, test temperature was varied to investigate the influence of kinetic viscosity of alkanes.

\section{Results and Discussion}

3.1. Signal Change Measurement Induced by Flying Instability. Figure 2 shows an example of VMM changes near the error position, indicated by red circle. Readback errors occurred at 2 sectors in a sequential read operation. No errors were detected in a sequential write operation. If the head cannot read the servodata and fails to set the positioning, the drive reports an error in a write operation. To determine if the errors were caused by a media magnetic defect or scratch the media was rewritten and VMM was remeasured. The VMM at the error positions recovered to normal value with rewriting, so it was concluded that there was no media magnetic defect or scratch present.

Figure 3 shows servogain and position error (PE) changes when abnormal servogain variation was detected in write operation. The large servogain means that the amplitude of the servo signal is small because automatic gain controller (AGC) adjusts the gain to maintain the signal level. When 


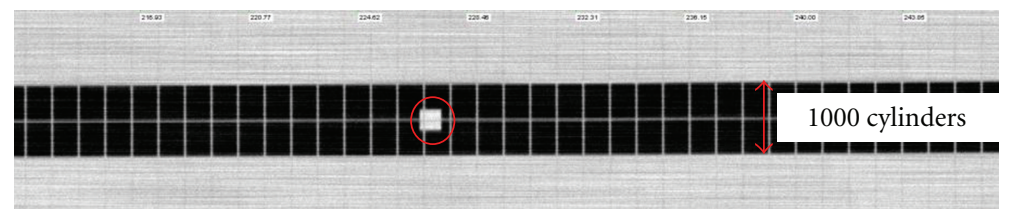

FIGURE 4: OSA image of media surface near the error location.
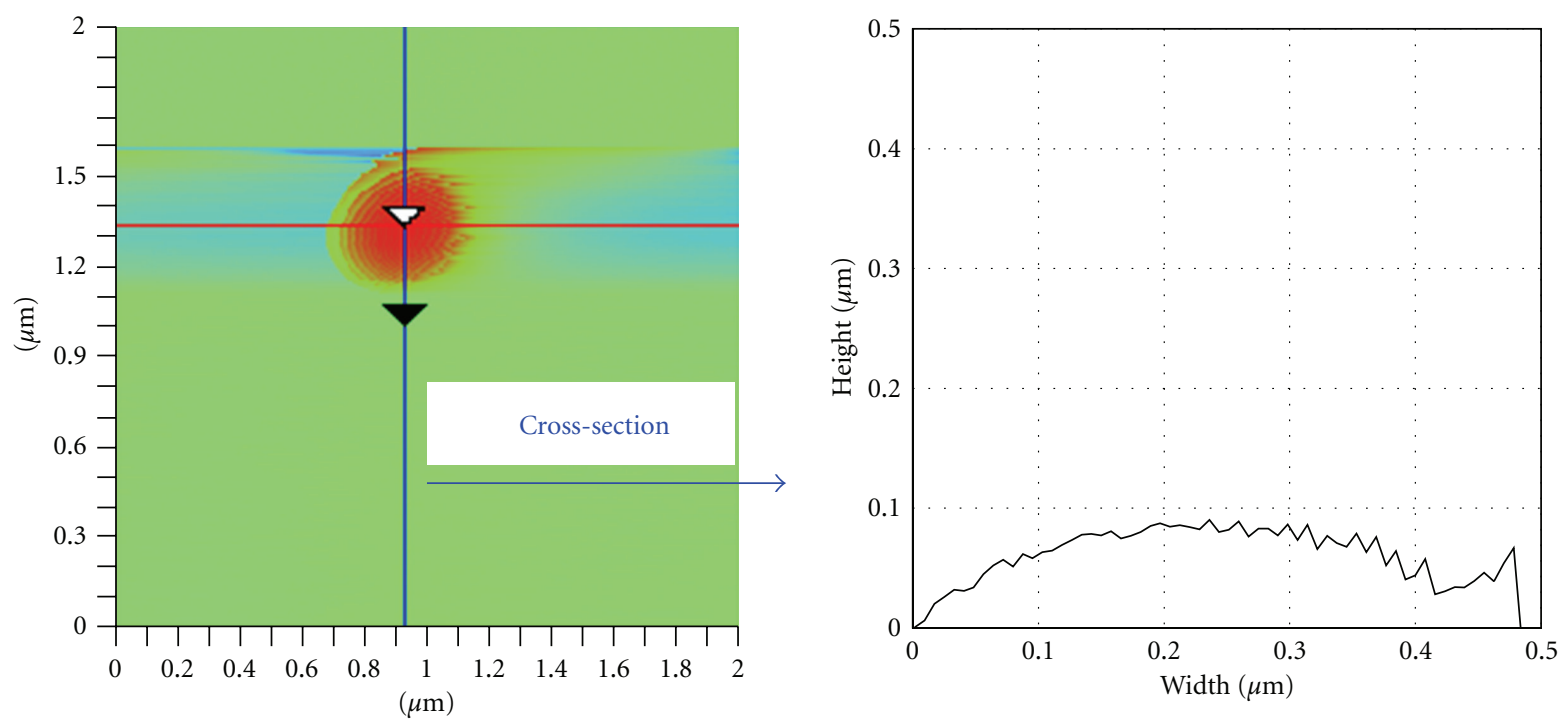

FIgURE 5: The droplet observation on the media by AFM.

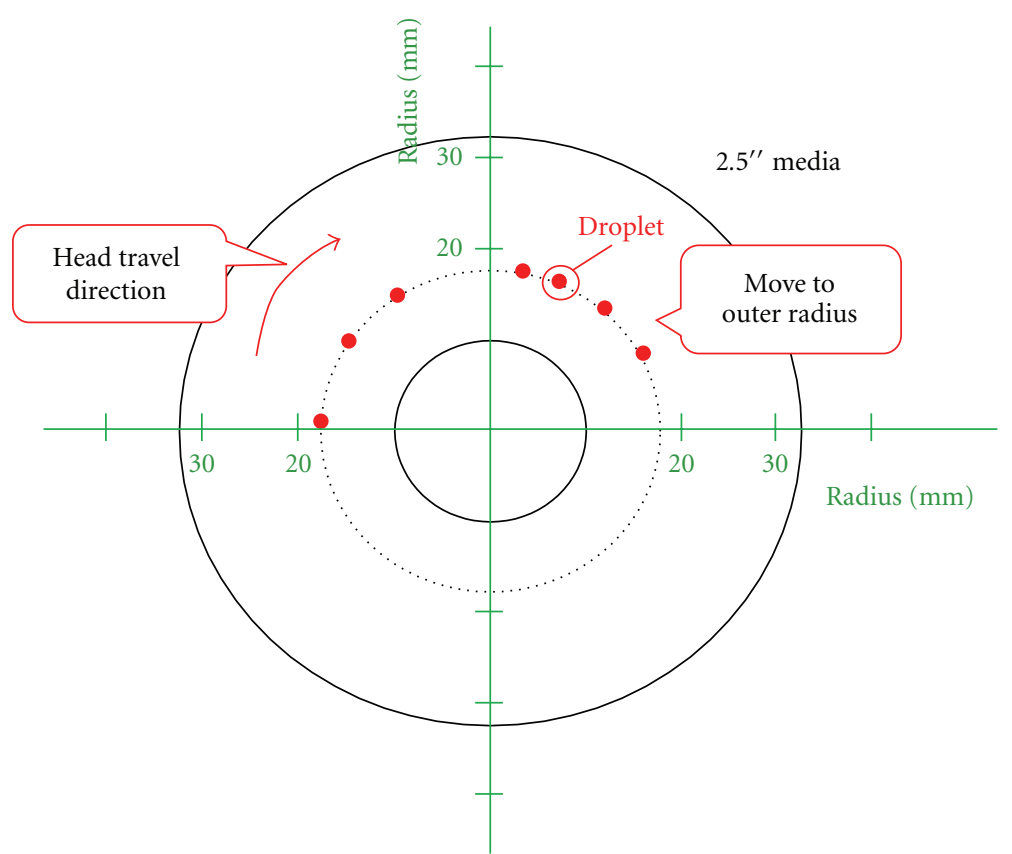

(a)

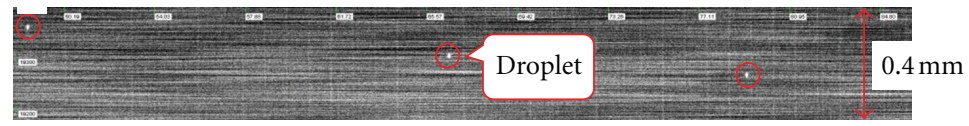

(b)

FIGURE 6: Droplet distribution map (a) and OSA image (b) on the media. The droplets in the down-track direction are located progressively further toward the disk outer radius, consistent with the head travel direction from inner to outer diameter. 


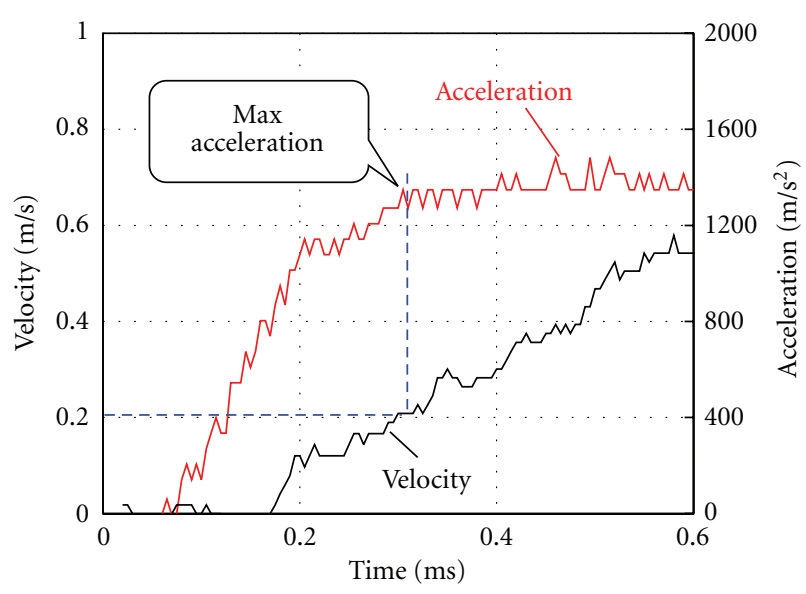

FIGURE 7: Radial velocity and acceleration of the head in full stroke seek operation.

the relative servogain changed from about 1.0 to 1.4 by flying instability, the flying height rise can be calculated to be around $7.5 \mathrm{~nm}$ using the Wallace spacing loss equation [4]. Although the $64 \mathrm{kHz}$ sampling frequency in this study is not large enough to identify specific ABS vibration modes, such as pitch and roll mode, the vibration frequency can be estimated roughly to be 5 to $10 \mathrm{kHz}$ which may correspond to that of suspension torsion mode.

On the other hand, PE was not seen to change significantly at the region where the servogain varied greatly. It indicates that the vibration does not move the cross-track direction, but vertical direction. The write operation was completed with no error being detected even though data could not be recorded correctly.

3.2. Droplet Observation. To further investigate the cause of the errors, we performed a drive teardown analysis and inspected the media and head surfaces for evidence of OC which may have caused the error. Before the drive teardown, the media was DC-erased around the error position to facilitate identification of the error location by an optical surface analyzer (OSA). No droplet was observed at the error location on the media surface indicated by red circle in Figure 4 . We speculate that the droplets were removed by contacts with head during write/read operations. To test this hypothesis, we measured the media surface from another drive after full stroke seek operation only. Based on full surface mapping of the media by OSA, several droplets were detected. Figure 5 shows one of the droplets measured by AFM. The shape of this droplet is dome-like. The height of this droplet is about $100 \mathrm{~nm}$, tall enough to make contact between the head and the droplet on the media.

Although we attempted to analyze the droplet by Auger electron spectroscopy and Raman spectroscopy, we did not succeed because the droplet vanished during their electron or laser irradiation inherent in the Auger and Raman techniques. Furthermore, some droplets disappeared when simply storing the media for a week at room temperature.
TABLE 1: Alkanes used for tests.

\begin{tabular}{lccccc}
\hline & Formula & $\begin{array}{c}\text { Volume } \\
(\mu \mathrm{L})\end{array}$ & $\begin{array}{c}\text { Density } \\
(\mathrm{g} / \mathrm{mL}) \\
\text { at } 20^{\circ} \mathrm{C}\end{array}$ & $\begin{array}{c}\text { Weight } \\
(\mathrm{mg})\end{array}$ & $\begin{array}{c}\mathrm{MP}^{*} \\
\left({ }^{\circ} \mathrm{C}\right)\end{array}$ \\
\hline Hexane & $\mathrm{C}_{6} \mathrm{H}_{14}$ & 100 & 0.70 & 70 & -95.0 \\
Hexadecane & $\mathrm{C}_{16} \mathrm{H}_{34}$ & & 0.77 & 77 & 18.0 \\
Icosane & $\mathrm{C}_{20} \mathrm{H}_{42}$ & 100 & 0.76 & 76 & 36.7 \\
Pentacosane & $\mathrm{C}_{25} \mathrm{H}_{52}$ & (Hexane & 0.80 & 80 & 53.0 \\
Triacontane & $\mathrm{C}_{30} \mathrm{H}_{62}$ & Solution) & 0.78 & 78 & 65.8 \\
\hline
\end{tabular}

Density data from International Chemical Safety Cards (ICSCs) or Material Safety Data Sheet (MSDS).

*MP: melting point.

These observations suggest that the droplets are volatile liquid.

Figure 6 shows the location of several droplets and the OSA image of representative droplets. The path of the droplets follows a spiral path toward the outer radius. A radial velocity of about $0.2 \mathrm{~m} / \mathrm{s}$ is calculated from the path. This velocity value indicates that the droplets dropped from head to media at the maximum acceleration in the full stroke seek operation, as shown in Figure 7.

Figure 8 shows an optical microscopy image of the head surface after the test. Some droplets were observed at the slider trailing edge and deposited ends. To identify whether the droplets are hydrocarbon or not, we analyzed these droplets on the head surface by Raman spectroscopy. The droplet on head surface was identified as hydrocarbon since the Raman spectrum of the droplet matched that of the reference hydrocarbon oil shown in Figure 9.

\subsection{The Influence of Kinetic Viscosity of Organic Compound.} Figure 10 shows the result of the alkane injection test. In general, melting point of higher molecular weight OC is higher as shown in Table 1. Therefore, lower molecular weight and high temperature make the OC more volatile. However, this result shows that higher molecular weight is prone to induce the error in spite of the amount of evaporated OC. In addition, for pentacosane (C25) time to failure of the test at lower temperature $\left(50^{\circ} \mathrm{C}\right)$ is shorter than the one at higher temperature $\left(75^{\circ} \mathrm{C}\right)$. It indicates that there may be another factor, distinct from the amount of gaseous OC causing the error.

Figure 11 shows examples of VMM changes near the error position or the position where VMM exceeds 3.8. Larger and longer VMM change occurred in the test for $\mathrm{C} 25$ at $50^{\circ} \mathrm{C}$ compared to other two tests (for $\mathrm{C} 25$ at $75^{\circ} \mathrm{C}$ and for $\mathrm{C} 16$ at $66^{\circ} \mathrm{C}$ ). That may be one of the reasons why the droplets at the test for $\mathrm{C} 25$ at $50^{\circ} \mathrm{C}$ have higher viscosity or larger size for. On the other hand, VMM changes also appeared in the other two tests in Figure 11 even if no error occurred. This implies the existence of the droplets at the three tests in Figure 11. From these results, we inferred that the higher kinetic viscosity associated with lower temperature and higher molecular weight or volume of alkane droplets are one of the significant factors which cause drive failure. To clarify the relationship between the kinetic 


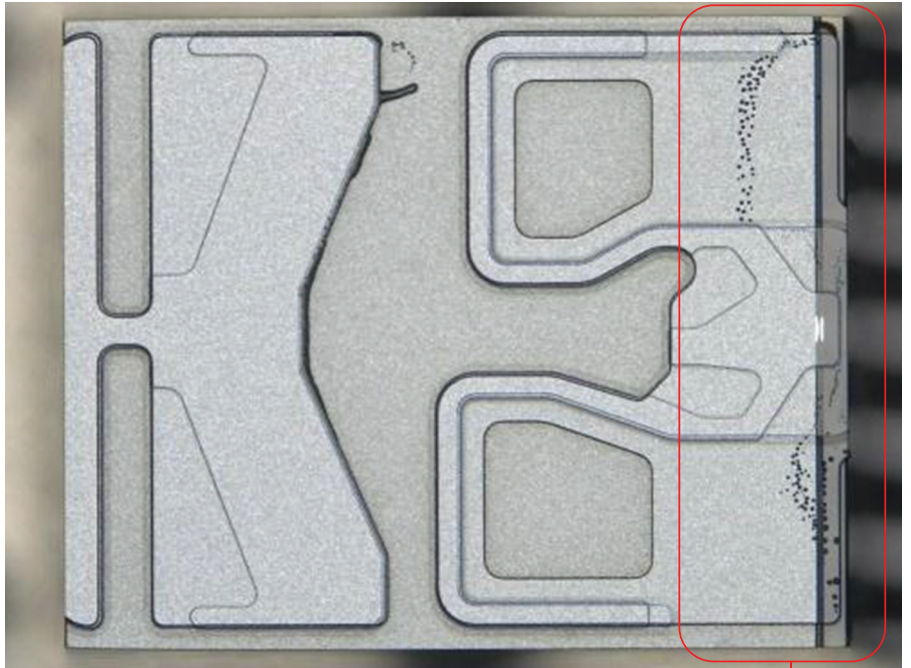

(a)

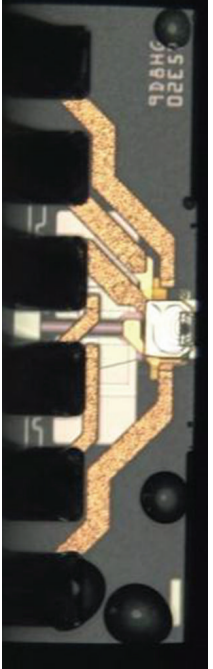

(b)

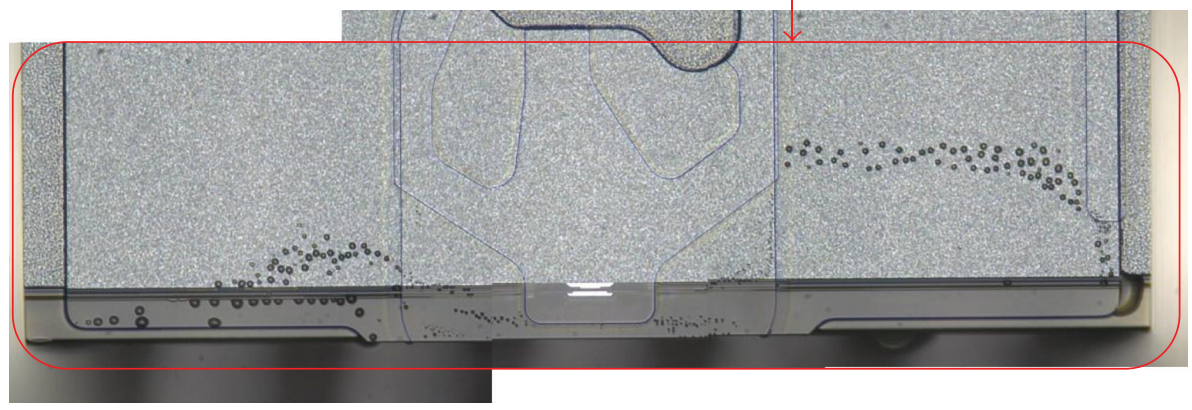

(c)

FIGURE 8: Optical microscope images (a) overall picture of ABS; (b) deposited ends of head; (c) enlarged picture of red square area in (a).

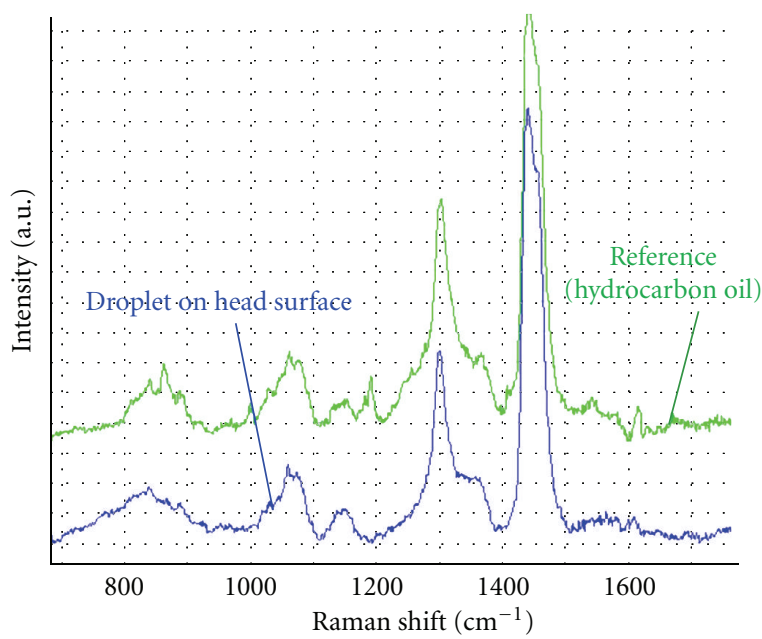

FIGURE 9: Droplet analysis by Raman spectroscopy.

viscosity of alkanes and occurrence of drive failure, plots of the test results were made as shown in Figure 12. We deduce from Figure 12 that there is a critical kinetic viscosity of OC for inducing drive failure, and the critical kinetic viscosity

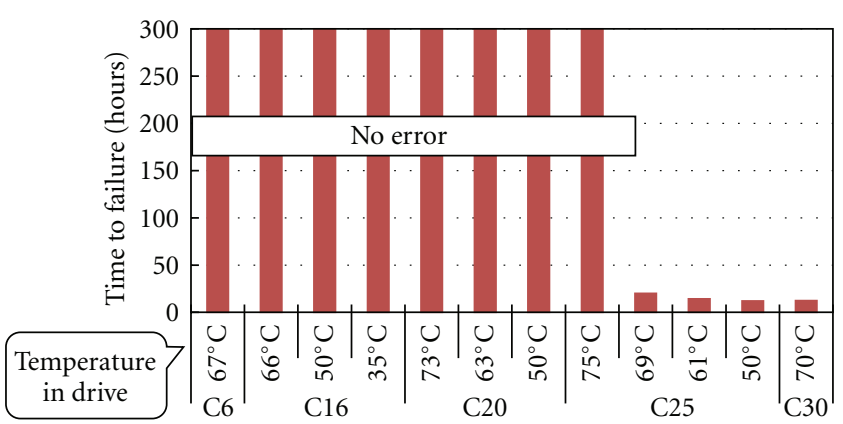

Figure 10: Alkane injection test result. The tests over $300 \mathrm{~h}$ were truncated.

is about $5 \mathrm{cSt}$ in the test. However, we need further study to separate from the droplet size (or volume) influence on inducing drive failure.

\section{Conclusion}

We studied the drive failure caused by the contact between the droplet of OC (hydrocarbon) and head. From our 

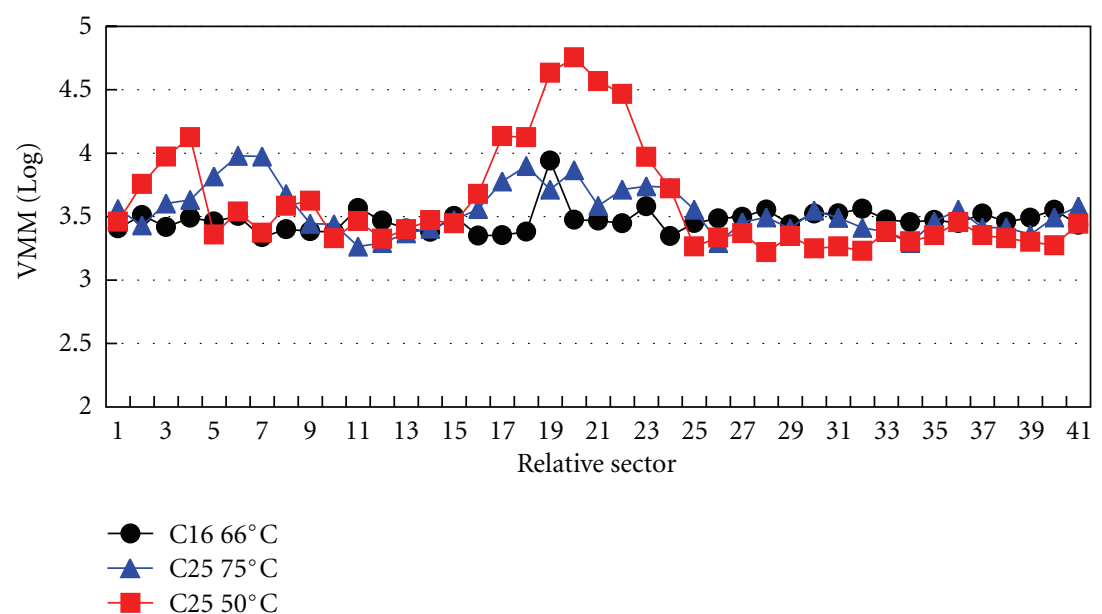

FIGURE 11: VMM change comparison near the error position $\left(\mathrm{C} 2550^{\circ} \mathrm{C}\right)$ or the position where $\mathrm{VMM}$ exceeds $3.8\left(\mathrm{C} 1666^{\circ} \mathrm{C}, \mathrm{C} 2575^{\circ} \mathrm{C}\right)$ in the alkane injection tests.

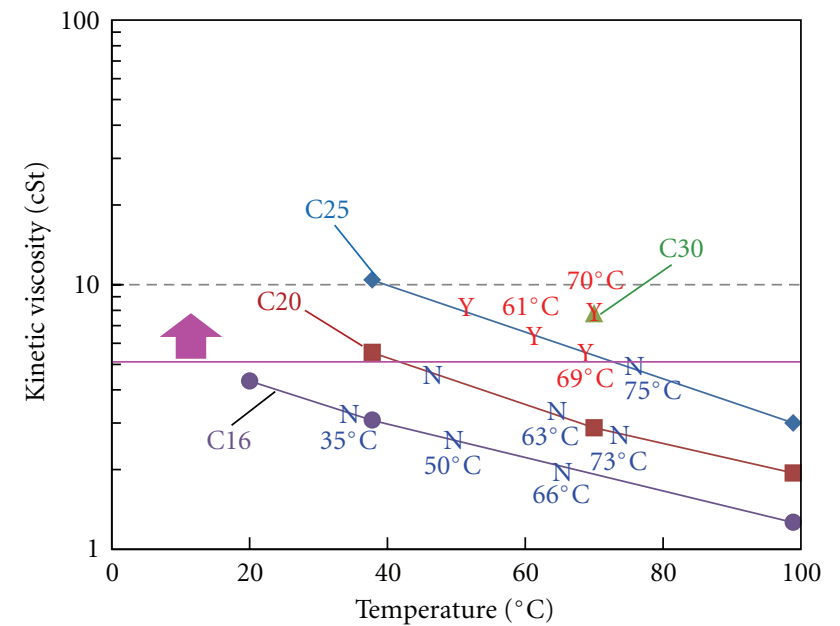

Y: error occurred

N: no error occurred

FIGURE 12: Correlation between kinetic viscosity of alkanes and the occurrence of drive errors.

investigations, we speculate the mechanism of the flying instability induced the OC as follows.

(1) OC evaporated from inside top cover condenses into a droplet on the head due to the pressurization under $\mathrm{ABS}$, or is adsorbed to disk surface. The OC adsorbed on the disk surface is picked up to the head surface then, the droplet is generated by accumulating the picked up OC.

(2) Droplets on the head transfer to the media by inertia force as the head seeks over the media.

(3) A large vibration occurs due to contact between the head and the droplet in the case of high kinetic viscosity components of OC or forming large droplet of OC.

\section{References}

[1] J. Li, J. Xu, and Y. Aoki, "Simulation on contact between the droplet and the slider at head-disk interface based on waterhammer pressure model," Microsystem Technologies, vol. 16, no. 1-2, pp. 57-65, 2010.

[2] D. E. Fowler and R. H. Geiss, "Chemical contamination at the head-disk interface in a disk drive," IEEE Transactions on Magnetics, vol. 36, no. 1, pp. 133-139, 2000.

[3] K. Aruga, "Probabilistic analysis of off-track capability assuming geometric track misregistration model for higher track density disk drives," IEEE Transactions on Magnetics, vol. 45, no. 11, pp. 5022-5025, 2009.

[4] R. L. Wallace, "The reproduction of magnetically recorded signal," Bell System Technical Journal, vol. 30, pp. 1145-1173, 1951. 

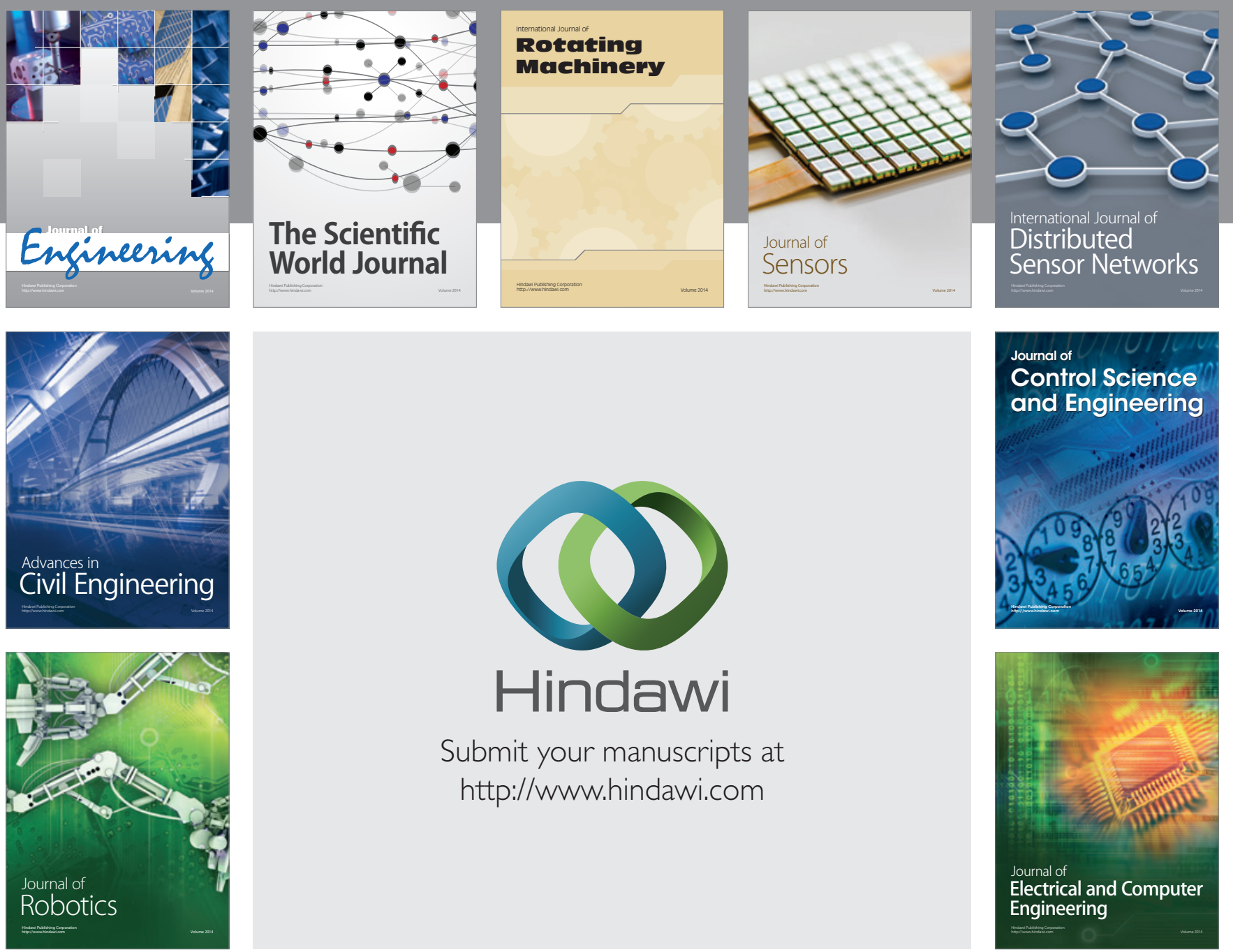

Submit your manuscripts at

http://www.hindawi.com
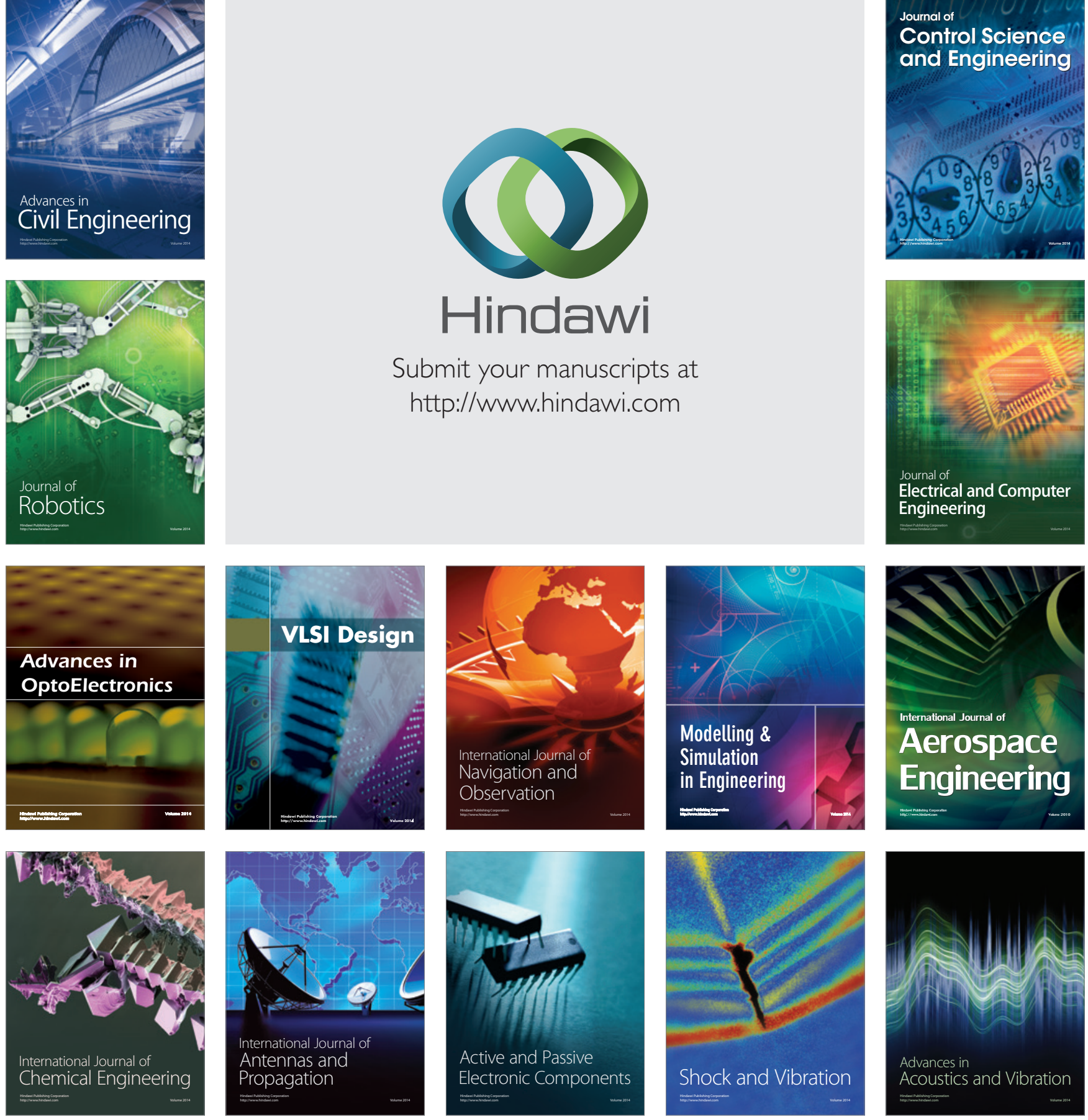